\title{
双半球型胶囊机器人弯曲肠道内视觉导航方法 ${ }^{*}$
}

\author{
张永顺 田 丰王智博 杨慧远 刘 旭 \\ (大连理工大学精密与特种加工教育部重点实验室 大连 116024)
}

\begin{abstract}
摘要: 为了实现主被动模态双半球型胶囊机器人在肠道内转弯行走, 提出了一种视觉导航方法, 首先利用动态调姿模态时胶 囊轴线与万向旋转磁场轴线的同轴随动特性, 并结合同一俯仰角和两个不同侧摆角下单目摄像头所拍摄的两幅无线传输图像 确定摄像头的姿态, 然后在摄像机坐标系内提取摄像头对准弯曲肠道时图像的暗区质心像素位置, 在万向均匀旋转磁场条件 下结合摄像头的姿态信息, 通过计算固定坐标系下弯曲肠道图像暗区质心的方位来确定胶囊的视觉导航方位, 最终确定胶囊 转弯滚动磁场轴线方向, 以便实现胶囊在肠道内的视觉辅助导航作业。试验证明该方法可用于胶囊导航, 不需要附加传感装 置, 胶囊结构紧凑, 为实现胃肠环境内遍历检查与医疗作业奠定了基础。
\end{abstract}

关键词: 双半球型胶囊机器人; 万向旋转磁场；姿态确定；暗区质心；视觉导航方位

中图分类号: TP242

\section{Visual Navigation Method of Dual Hemisphere Capsule Robot inside Curved Intestine Tract}

\author{
ZHANG Yongshun TIAN Feng WANG Zhibo YANG Huiyuan LIU Xu \\ (Key Laboratory for Precision \& Non-traditional Machining of Ministry of Education, \\ Dalian University of Technology, Dalian 116024)
}

\begin{abstract}
To realize the steering locomotion of the dual hemisphere capsule robot with active and passive modes in curved intestinal tract, a visual navigation method is proposed. First, based on the coaxial follow-up characteristics of the capsule axis and the spatial universal rotating magnetic vector during dynamic posture adjustment process, the posture of the embedded monocular camera can be calculated by its two wireless transmission images taken at the same altitude angle but two different azimuth angles. Then, the centroid pixel coordinates of the dark region of the image can be extracted in the camera coordinate when the camera is aligned with the curved intestine, and under the condition of uniform spatial universal rotating magnetic field and by combination with the posture of the camera, the direction of visual navigation of the capsule is derived by calculating the orientation of the centroid of the dark region of the image in the fixed coordinate system, thus the visual navigation of the capsule in the curved intestine tract can be achieved by further calculating the direction of the the spatial universal rotating magnetic vector for rolling locomotion. The test proves that the method can be used for capsule navigation without any additional sensor device, with more effective load volume, the navigation method lays a foundation for all-over inspection and medical operation inside the gastrointest-inal tract of human body.
\end{abstract}

Key words: dual hemisphere capsule robot; spatial universal rotating magnetic field; determination of posture information; centroid of dark region; direction of visual navigation

\section{0 前言}

胃肠道容易发生一些致命性疾病 ${ }^{[1]}$ ，早期诊断 非常重要, 最常用的器械是传统内窥镜, 由于导管 可插入长度有限, 无法对整个肠道进行检查。且容

* 国家自然科学基金资助项目(61773084)。20190401 收到初稿, 20191224 收到修改稿
易造成胃肠道软组织损伤, 甚至引起患者痛苦 ${ }^{[2]}$ 。

为实现胃肠道的无创遍历诊疗, 无线胶囊内窥 镜实现了从有线到无线的突破, 主要包括被动式和 外磁场驱动胶囊内窥镜, 前者在体内行走依靠胃肠 蠕动和重力或者自身行走机构, 无法实现行走和姿 态的主动控制, 诊断结果可靠性低; 后者驱动主要 依靠外磁场驱动实现胶囊内窥镜位置与姿态的控 制, 但运动与姿态控制相互影响, 不能实现定点观 
察, 尤其在结肠等宽裕环境内姿态控制困难, 漏 检率较高 ${ }^{[3-5]}$ 。事实上, 外部磁场驱动胶囊机器人 已经成为研究主流 ${ }^{[6]}$ 。大连理工大学提出一种由 空间万向旋转磁场驱动的双半球型胶囊机器人及 其转弯驱动控制方法 ${ }^{[7-8]}$ 。该机器人适用于结肠等 宽裕环境内操作, 解决了动态姿态调整与滚动行 走两种模态相互分离的关键技术, 实现了胶囊的 定点动态调姿。

然而, 双半球型胶囊进入人体后, 摄像机的位 置和姿态信息未知, 即使借助实时视频辅助也无法 确定导航方位, 目前胶囊的转弯导航只能借助图像 通过多次尝试方法调整胶囊姿态直到近似对准肠道 弯曲方向 ${ }^{[9]}$, 可操作性差, 精度低且效率不高。为 了提高胶囊转弯性能, 必须解决胶囊弯曲方向的导 航问题。

目前胶囊内窥镜领域常用的导航装置有内置陀 螺仪或其他传感器组, 但受限于胶囊体积 ${ }^{[10-11]}$; 磁 阵列法会受万向旋转磁场的干扰 ${ }^{[12-13]}$; 多目摄像机 导航系统需要高安装精度配合且体积过大 ${ }^{[14-15]}$; 复 杂肠道三维重建技术和基于暗区或纹理的肠道导航 方法都缺少胶囊和内嵌摄像机的姿态信息, 因此无 法确定具体的导航方位 ${ }^{[16-17]}$ 。本文提出一种单目视 觉辅助导航方法, 该方法首先利用胶囊轴线与万向 磁场旋转轴线的同轴随动特性, 结合同一俯仰角和 两个不同侧摆角下单目摄像机所拍摄的两幅无线 传输图像确定胶囊内嵌摄像机的姿态信息, 再通过 计算固定坐标系下弯曲肠道图像暗区质心的方位 来确定肠道弯曲方向, 实现胶囊在肠道内的视觉辅 助导航作业。最后通过离体猪大肠试验证明该导航 方法可有效的在弯曲肠道环境中确定胶囊的导航 方位, 该方法简单, 不需要附加传感定位装置, 为 实现胃肠道内遍历检查与定点靶向治疗等医疗作 业奠定了基础。

\section{1 胶囊结构及工作原理}

胶囊结构如图 1 所示, 包括主、被动半球体, 摄像头组件, $\mathrm{NdFeB}$ (钕铁硼)永磁体, 轴承, 轴, 套 筒。主动半球壳与其内部的套筒及径向磁化 $\mathrm{NdFeB}$ 永磁体固结; 被动半球壳与其内部的轴和摄像头组 件固结, 主、被动半球体由轴承悬浮连接, 可相对 转动。主动半球由耦合磁矩直接驱动转动, 被动半 球无磁驱动源, 处于欠驱动状态。

主要结构参数如表 1 所示。基于双半球特殊结 构, 胶囊能实现悬停调姿和滚动行走两个工作状态。 悬停调姿状态又可称为被动模态, 滚动行走状态为
主动模态。两个模态可通过调整外磁场的方向导引 来实现。当胶囊处于被动模态时, 在自站立特性作 用下, 被动半球与肠壁接触, 胶囊实现定点悬停调 姿, 通过改变外加磁场的方向可任意调整胶囊姿态, 因此，摄像机可捕捉肠内不同角度图像。当胶囊需 要前进时, 可将胶囊切换至主动模态, 通过调整磁 场方向来控制胶囊运动方向 ${ }^{[18]}$ 。

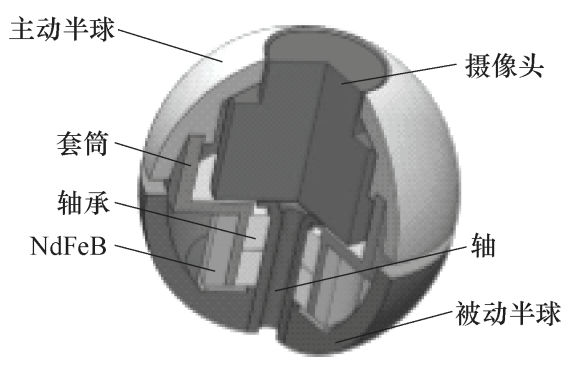

图 1 双半球胶囊机器人结构图

表 1 机器人主要结构参数

\begin{tabular}{|c|c|}
\hline 参数 & 数值 \\
\hline 机器人直径 $/ \mathrm{mm}$ & 20 \\
\hline 质量/g & 5 \\
\hline 球壳壁厚/mm & 1.5 \\
\hline 轴承尺寸 $\left(\frac{\text { 长 }}{\mathrm{mm}} \times \frac{\text { 宽 }}{\mathrm{mm}} \times \frac{\text { 高 }}{\mathrm{mm}}\right)$ & $6 \times 3 \times 2.5$ \\
\hline $\mathrm{NdFeB}$ 尺寸 $\left(\frac{\text { 长 }}{\mathrm{mm}} \times \frac{\text { 宽 }}{\mathrm{mm}} \times \frac{\text { 高 }}{\mathrm{mm}}\right)$ & $7.5 \times 6 \times 5$ \\
\hline 球壳材料 & 聚乳酸降解塑料 \\
\hline 套筒材料 & 铝合金 \\
\hline
\end{tabular}

\section{2 胶囊导航过程}

图 2 为肠道检查系统原理图。首先, 患者吞下 胶囊, 通过摄像头组件将视频信号实时传输到外部 接收设备, 然后操作者根据外部控制设备接收的实 时诊疗画面和计算的导航方向，通过手柄控制固定 坐标系内侧摆角 $\theta$ 与俯仰角 $\delta$ 两个参数, 从而控制 磁场轴线方向 $\boldsymbol{n}_{s}$, 决定继续定点 “悬停” 调整姿态 实现全方位观察或滚动改变观察点。诊疗结束后胶 囊随废物排出体外 ${ }^{[19]}$ 。

胶囊导航过程如图 3 所示。当胶囊在 $A$ 处需要 导航时, 此时胶囊轴线方向为 $\boldsymbol{n}_{1}$, 拍摄第一幅图像 并提取图像暗区质心; 保持磁场轴线方向的俯仰角 不变, 只改变侧摆角, 胶囊轴线方向变为 $\boldsymbol{n}_{2}$, 拍摄 第二幅图像并提取图像暗区质心，利用以上两幅图 像通过弯曲肠道内胶囊视觉导航方法先确定胶囊的 姿态信息,然后计算出从 $A$ 处到 $B$ 处的导航方向 $\boldsymbol{n}_{s}$ 并控制万向旋转磁场使胶囊轴线方向对准 $B$ 处, 最 后计算需要施加旋转磁矢量的法线方向 $\boldsymbol{n}_{4}$, 其中 $\boldsymbol{n}_{4}$ 
与导航方向 $\boldsymbol{n}_{s}$ 垂直, 控制胶囊从 $A$ 滚动到 $B$ 处。 在 $B$ 处重复以上步骤, 即可控制胶囊运动到 $C$ 处, 从而完成转弯过程。

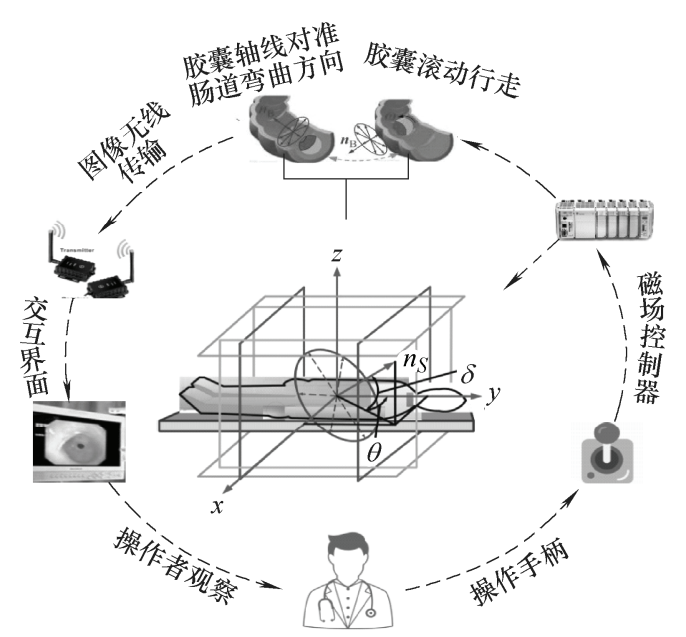

图 2 肠道检查系统原理图

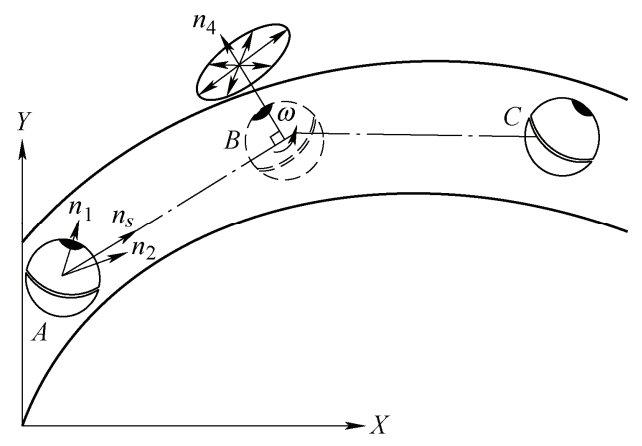

图 3 导航过程示意图

\section{3 摄像机姿态和暗区质心的确定}

由于摄像机与胶囊的被动半球固连, 姿态调 整时不旋转, 故摄像机姿态与胶囊姿态一致。利 用胶囊在旋转磁场中的随动效应, 可知胶囊轴线 始终与旋转磁矢量方向一致, 即摄像机的光轴方 向也与旋转磁矢量方向一致, 因此, 姿态调整后 胶囊静止时摄像机的光轴方向已知, 可用固定坐 标系内侧摆角 $\theta$ 与俯仰角 $\delta$ 两个姿态角描述, 但 摄像机框架绕胶囊轴线的自转角度未知, 并具有 随机性, 因此, 无法确定摄像机姿态, 还需要确 定摄像机绕胶囊轴线的自转角信息才能确定摄像 机姿态。

确定摄像机绕胶囊轴线自转角的方法为: 当胶 囊调姿完成处于静止时, 此时拍摄图像画面为 $V_{1}$, $V_{1}$ 上的肠道暗区质心点为 $d_{1}$; 保持磁矢量方向的俯 仰角不变, 只改变侧摆角, 胶囊再次处于静止, 此 时图像画面为 $V_{2}, V_{2}$ 上的肠道暗区质心点为 $d_{2}$ 。侧
摆过程中胶囊主动半球空转, 摄像机绕胶囊轴线的 自转角保持不变。设操控侧摆角 $V_{1}$ 逆时针变化为正, 从摄像机光心沿光轴方法观察像平面, 记像平面绕 光轴顺时针旋转为正。

当操控侧摆角 $V_{1}$ 逆时针变化, 像平面绕胶囊轴 线旋转 $0 \sim 90^{\circ}$ 时, $V_{1}$ 与 $V_{2}$ 的关系如图 4a 所示, 将 点 $d_{2}$ 的像素坐标变换为 $V_{1}$ 上的点 $d_{2}^{\prime}, \boldsymbol{d}_{2}^{\prime} \boldsymbol{d}_{1}$ 的连线与 水平面平行, 过 $d_{1}$ 作直线平行于像平面上边缘线, 该直线与矢量 $\boldsymbol{d}_{2}^{\prime} \boldsymbol{d}_{1}$ 所夹锐角 $\alpha$ 即为像平面绕胶囊轴 线的自转角 $\alpha^{*}$ 。因此 $\alpha^{*}$ 可通过点 $d_{1}, d_{2}$ 的像素坐标 计算求得。

当操控侧摆角逆时针变化, 像平面绕胶囊轴线 旋转 $-90^{\circ} \sim 0$ 时, 如图 $4 \mathrm{~b}$ 所示, 此时像平面绕胶囊 轴线的自转角 $\alpha^{*}=-\alpha$ 。

当操控侧摆角逆时针变化, 像平面绕胶囊轴线 旋转 $90^{\circ} \sim 180^{\circ}$ 时, 如图 4c 所示, 此时像平面绕胶 囊轴线的自转角 $\alpha^{*}=90^{\circ}+90^{\circ}-\alpha=180^{\circ}-\alpha^{\circ}$ 。

当操控侧摆角逆时针变化, 像平面绕胶囊轴线 旋转 $-180^{\circ} \sim-90^{\circ}$ 时, 如图 $4 \mathrm{~d}$ 所示, 此时像平面绕 胶囊轴线的自转角 $\alpha^{*}=-\left(90^{\circ}+90^{\circ}-\alpha\right)=\alpha-180^{\circ}$ 。

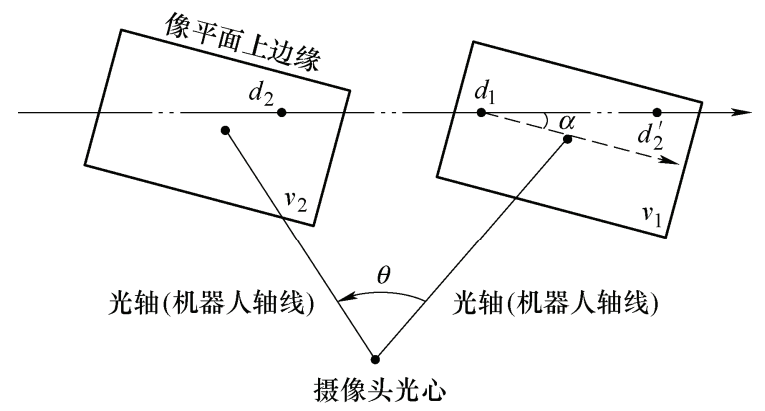

(a) 自转角 $0 \sim 90^{\circ}$ 区域内

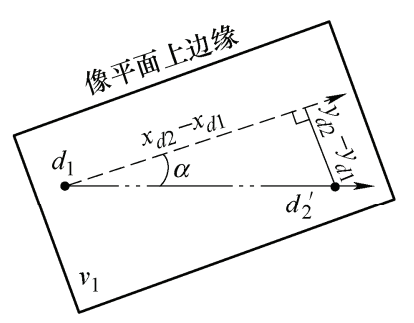

(b) 自转角 $0 \sim-90^{\circ}$ 区域内

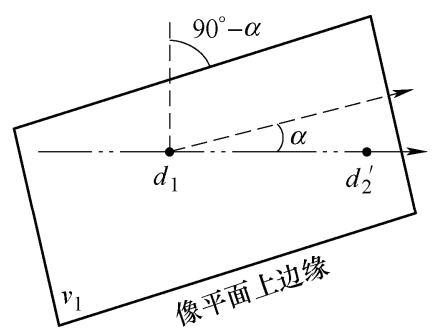

(c) 自转角 $90^{\circ} \sim 180^{\circ}$ 区域内 


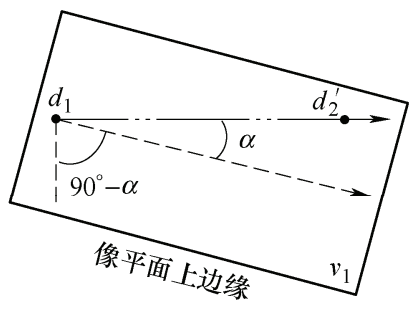

(d) 自转角 $-90^{\circ} \sim-180^{\circ}$ 区域内

图 4 侧摆角 $\theta$ 逆时针顺序获取两幅连续图像关系示意图

当操控侧摆角顺时针变化, 情况与侧摆角逆时 针变化完全相反。操控侧摆角顺时针或逆时针变化, 可按自转角度分成以上四种情况, 可通过 $d_{1}, d_{2}$ 的 像素坐标代数关系判断此时像平面的所属情形。将 所有情形及判断方法总结如下。

当操控侧摆角逆时针变化时, 像平面绕胶囊轴 线的自转角度

$$
\alpha^{*}=\frac{\left(y_{d 1}-y_{d 2}\right)}{\left|y_{d 1}-y_{d 2}\right|} \cdot \arccos \frac{\left|x_{d 2}-x_{d 1}\right|\left(x_{d 2}-x_{d 1}\right)}{\sqrt{l_{1}} \cdot \sqrt{l_{2}}}
$$

当操控侧摆角顺时针变化时, 像平面绕胶囊轴 线的自转角度

$$
\begin{gathered}
\alpha^{*}=\frac{\left(y_{d 2}-y_{d 1}\right)}{\left|y_{d 2}-y_{d 1}\right|} \cdot \arccos \frac{\left|x_{d 1}-x_{d 2}\right|\left(x_{d 1}-x_{d 2}\right)}{\sqrt{l_{1}} \cdot \sqrt{l_{2}}} \\
\left\{\begin{array}{l}
l_{1}=\left(x_{d 2}-x_{d 1}\right)^{2}+\left(y_{d 2}-y_{d 1}\right)^{2} \\
l_{2}=\left(x_{d 2}-x_{d 1}\right)^{2}
\end{array}\right.
\end{gathered}
$$

式中, $x_{d 1}, y_{d 1}, x_{d 2}, y_{d 2}$ 分别为 $d_{1}, d_{2}$ 点的像素横、纵 坐标。

在校正自转角之后, 需要确定弯曲肠道的暗区质 心。本文提出了基于改进的 Bat Algorithm(BAT)算 法 ${ }^{[20]}$, 结合二维 OTSU 算法相结合的暗区质心识别方 法, 能在一定程度提高肠道内暗区识别的准确性, 也 能改善算法的实时性问题。在 BAT 算法中并没有关于 局部挖掘能力与全局探索能力的设计, 因此本文在传 统的 BAT 算法中加入自适应速度系数 $H$ 。自适应速度 系数意味着上一代搜索的结果会对当前代的搜索速度 产生影响。控制 $H$ 取值即可调节阈值计算过程中的各 个阶段寻优范围。图 5 为提取暗区质心的算法流程图。

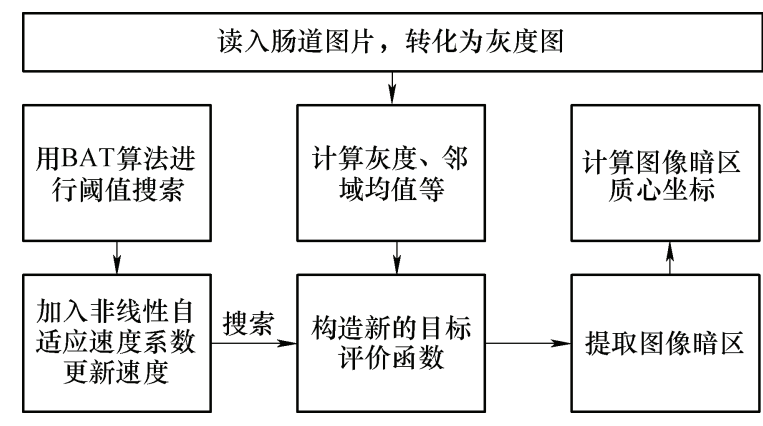

图 5 算法流程图

\section{4 导航方向的确定}

当胶囊进入工作环境时, 一般需要获知其在体 内的位置及姿态才能进行导航。图 6 为三轴亥姆霍 兹线圈固定坐标系 $O X Y Z$ 与机器人摄像机光心 $O_{C}$ 固连动坐标系 $O_{C} X_{W} Y_{W} Z_{W}$ 间的对应关系, 由于三轴 亥姆霍兹线圈产生的旋转磁场具有区域均匀性及各 点同向性, 所以在磁场均匀区内, 无论胶囊处于任 何位置, 其轴线方向始终与磁矢量方向一致, 因此 可将固定坐标系 $O X Y Z$ 的原点 $O$ 平移至摄像机的光 心 $O_{C}$, 坐标轴方向不变, 构成动坐标系 $O_{C} X_{W} Y_{W} Z_{W}$ 。

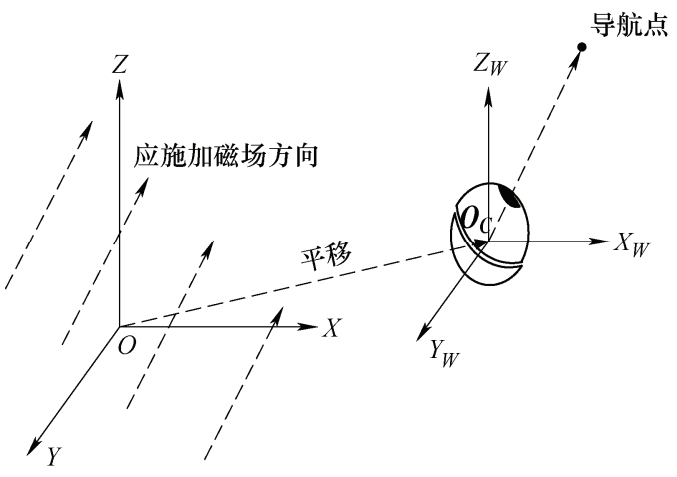

图 6 固定坐标系与动坐标系关系

所要求解的固定坐标系内导航点相对于胶囊的 方向矢量等价于导航点在动坐标系 $O_{C} X_{W} Y_{W} Z_{W}$ 内 相对于 $O_{C}$ 的方向矢量。因此只需要计算出导航点在 动坐标系中相对于原点的方向矢量, 此矢量即导航 方向。故我们只需要根据式(1)、(2)求得胶囊的姿态 信息, 并不需要获知胶囊的位置信息即可借助图像 实现导航。坐标系的变换关系如图 7 所示, 包含固 定坐标系 $O X Y Z$ 、动坐标系 $O_{C} X_{W} Y_{W} Z_{W}$ 、胶囊轴线 坐标系 $O_{C} X_{1} Y_{1} Z_{1}$ 、摄像机轴线坐标系 $O_{C} X_{C} Y_{C} Z_{C}$ 、 图像像素坐标系 $O_{0} u v$ 和图像物理坐标系 $O_{1} x y$, 其中 胶囊和相机的轴线坐标系 $Z$ 轴经过摄像机光心, 两 坐标系之间存在沿 $Z$ 轴的自转角度 $\alpha^{*}$ 。动坐标系 $O_{C} X_{W} Y_{W} Z_{W}$ 和胶囊轴线坐标系 $O_{C} X_{1} Y_{1} Z_{1}$ 的对应关 系如图 8 所示, 将动坐标系先绕 $O_{C} Z_{W}$ 轴顺时针旋 转 $\theta$ 度, 再绕 $O_{C} X_{W}$ 轴逆时针旋转 $\delta$, 得到胶囊轴 线坐标系 $O_{C} X_{1} Y_{1} Z_{1}$, 此时胶囊轴线方向与 $O_{C} Z_{1}$ 轴 重合; 胶囊轴线坐标系 $O_{C} X_{1} Y_{1} Z_{1}$ 绕 $O_{C} Z_{1}$ 轴顺时针 旋转 $\alpha^{*}$ 角度可得到摄像机轴线坐标系 $O_{C} X_{C} Y_{C} Z_{C}$; 图像物理坐标系 $O_{1} x y$ 垂直于 $O_{C} Z_{C}$ 轴, 原点 $O_{1}$ 在 $O_{C} Z_{C}$ 轴上, $O_{1} O_{C}$ 的长度为摄像机焦距 $f$, 且 $O_{1} \mathrm{x}$ 轴 与 $O_{C} Z_{C}$ 轴平行, $O_{1} y$ 轴与 $O_{C} Y_{C}$ 轴平行; 图像像素 坐标系 $O_{0} u v$ 和图像物理坐标系 $O_{1} x y$ 对应关系如 图 9 所示, 图像物理坐标系原点 $O_{1}$ 向 $y$ 轴负方向平 
移 $v_{0}$, 再向 $x$ 轴负方向平移 $u_{0}$, 得到图像像素坐 标系 $O_{0} u v$ 。

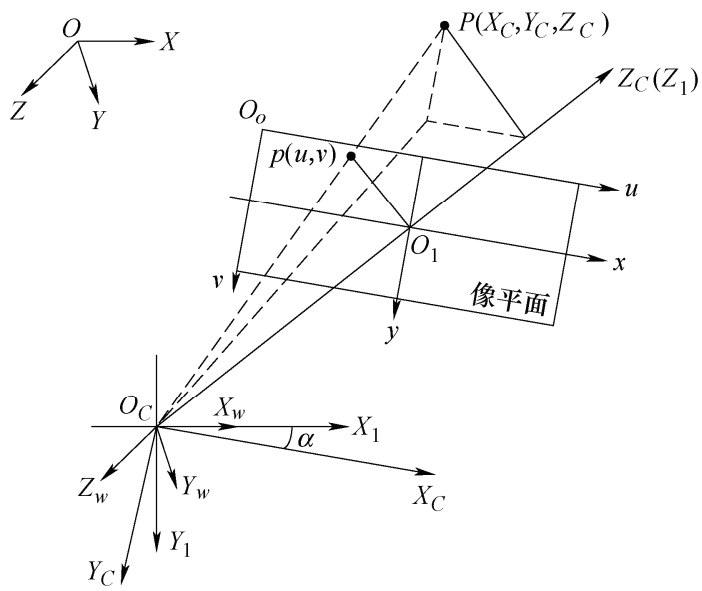

图 7 坐标系变换关系

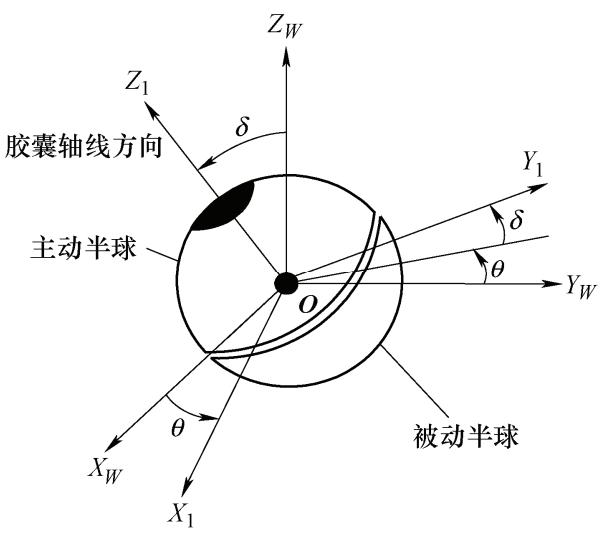

图 8 动坐标系和胶囊轴线坐标系变换关系

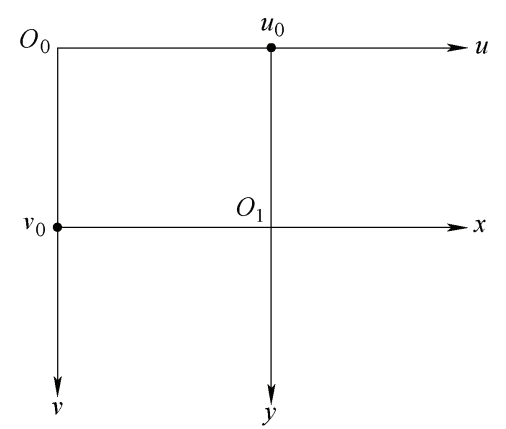

图 9 图像像素坐标系与物理坐标系关系

设已知胶囊在旋转磁场作用下, 当前磁矢量方 向侧摆角为 $\theta$, 俯仰角为 $\delta$, 摄像机绕胶囊轴线的 自转角度 $\alpha^{*}, p(u, v)$ 为图像像素坐标系上的暗区质 心坐标点, $P$ 点是与 $p$ 对应的物点, 即目标导航点。 所要求的最终导航方向即导航点在动坐标系中相对 于原点的方向矢量 $\boldsymbol{O}_{C} p$ 与 $\boldsymbol{O}_{C} \boldsymbol{P}$ 的方向一致, 因此确 定导航方向时, 需要将图像中点 $p(u, v)$ 的表示从像 素坐标系转化成动坐标系 $O_{C} X_{W} Y_{W} Z_{W}$, 先获得图像 物理坐标和图像像素坐标系之间的关系

$$
\left(\begin{array}{l}
x \\
y \\
f
\end{array}\right)=\left(\begin{array}{ccc}
\mathrm{d} x & 0 & -u_{0} \mathrm{~d} x \\
0 & \mathrm{~d} y & -v_{0} \mathrm{~d} y \\
0 & 0 & f
\end{array}\right)\left(\begin{array}{l}
u \\
v \\
1
\end{array}\right)
$$

式中, $\mathrm{d} x, \mathrm{~d} y$ 为像元尺寸, $u_{o}, v_{o}$ 为图像中心在像平 面的位移, $f$ 为摄像机焦距。

摄像机轴线坐标系内的点 $(x, y, f)$ 转换到胶囊 轴线坐标系 $O_{C} X_{1} Y_{1} Z_{1}$ 的矩阵为

$$
\left(\begin{array}{l}
x \\
y \\
f
\end{array}\right)=\left(\begin{array}{ccc}
\cos \alpha^{*} & \sin \alpha^{*} & 0 \\
-\sin \alpha^{*} & \cos \alpha^{*} & 0 \\
0 & 0 & 1
\end{array}\right)\left(\begin{array}{l}
X_{1} \\
Y_{1} \\
f
\end{array}\right)
$$

逆运算得

$$
\left(\begin{array}{l}
X_{1} \\
Y_{1} \\
f
\end{array}\right)=\left(\begin{array}{ccc}
\cos \alpha^{*} & -\sin \alpha^{*} & 0 \\
\sin \alpha^{*} & \cos \alpha^{*} & 0 \\
0 & 0 & 1
\end{array}\right)\left(\begin{array}{l}
x \\
y \\
f
\end{array}\right)
$$

最后将 $\left(X_{1}, Y_{1}, f\right)$ 转换到动坐标系 $O_{C} X_{W} Y_{W} Z_{W}$ 中, 正交变换矩阵为

$$
\boldsymbol{A}=\left(\begin{array}{ccc}
\cos \theta & -\sin \theta & 0 \\
\sin \theta \cos \delta & \cos \theta \cos \delta & \sin \delta \\
-\sin \theta \sin \delta & -\cos \theta \sin \delta & \cos \delta
\end{array}\right)
$$

则

$$
\left(\begin{array}{c}
X_{1} \\
Y_{1} \\
f
\end{array}\right)=\boldsymbol{A} \cdot\left(\begin{array}{c}
X_{W} \\
Y_{W} \\
Z_{W}
\end{array}\right)
$$

结合以上坐标变换, 即可计算图像暗区质心在 动坐标系 $O_{C} X_{W} Y_{W} Z_{W}$ 内的方向 $\boldsymbol{n}_{s}$

$$
\begin{gathered}
\left(\begin{array}{c}
X_{W} \\
Y_{W} \\
Z_{W}
\end{array}\right)=\boldsymbol{A}^{-1} \boldsymbol{Q}_{1} \boldsymbol{Q}_{2} \cdot\left(\begin{array}{l}
u \\
v \\
1
\end{array}\right) \\
\boldsymbol{Q}_{1}=\left(\begin{array}{ccc}
\cos \alpha^{*} & -\sin \alpha^{*} & 0 \\
\sin \alpha^{*} & \cos \alpha^{*} & 0 \\
0 & 0 & 1
\end{array}\right) \\
\boldsymbol{Q}_{2}=\left(\begin{array}{ccc}
\mathrm{d} x & 0 & -u_{0} \mathrm{~d} x \\
0 & \mathrm{~d} y & -v_{0} \mathrm{~d} y \\
0 & 0 & f
\end{array}\right)
\end{gathered}
$$

再通过正交变换方法 ${ }^{[21]}$, 将 $\boldsymbol{n}_{s}$ 用侧摆角 $\theta$ 和俯 仰角 $\delta$ 两个变量表示, 即得到了视觉导航方位。由 于滚动磁场需在水平面内施加, 因此, 将视觉导航 方位的 $\delta$ 值设为 0 , 即得到了导航应施加的滚动磁 场, 由此完成胶囊的导航。

\section{5 试验验证}

为了避免万向旋转磁场误差和胶囊动态姿态调 
整稳定性对导航试验精度的影响, 设计试验装置如 图 10a 所示, 包括胶囊姿态模拟装置 $\mathrm{A}$, 外部接收 装置 $\mathrm{B}$ 以及一段离体猪大肠。试验采用计算机 $\mathrm{CPU}$ 型号为 Intel G530, 内存为 $4 \mathrm{~GB}$, Windows $7 \times 64$ 位 操作系统, Matlab(R2014a), 其中相机的内部参数 为, 焦距 $3.7 \mathrm{~mm}$, 广角 $60^{\circ}$, 图像中心 $v_{0}$ 为 $240 \mathrm{~mm}$, $u_{0}$ 为 $320 \mathrm{~mm}$, 像元尺寸 $d_{x}$ 为 $466.14 \mathrm{~mm}, d_{y}$ 为 $466.14 \mathrm{~mm}$ 。

装置 $\mathrm{A}$ 的内部结构如图 $10 \mathrm{~b}$ 所示, 将肠道固定 装置固定在水平面内, 万向球型胶囊模拟装置在 肠道固定装置内可实现侧摆, 俯仰转动及绕姿态 控制端轴线的自转, 因此, 可通过操控姿态控制 端控制万向球型胶囊模拟装置在肠道固定装置内 的姿态来模拟胶囊定点调姿时 $\theta, \delta, \alpha^{*}$ 的变化。万 向球型胶囊模拟装置的俯仰角、侧摆角、自转角 轴线姿态信息可由三维姿态传感器读出。摄像机 拍摄的视频通过无线视频传输电路发射, 由装置 $\mathrm{B}$ 中的外部无线接收器接收, 再由视频转换器接 入计算机显示。

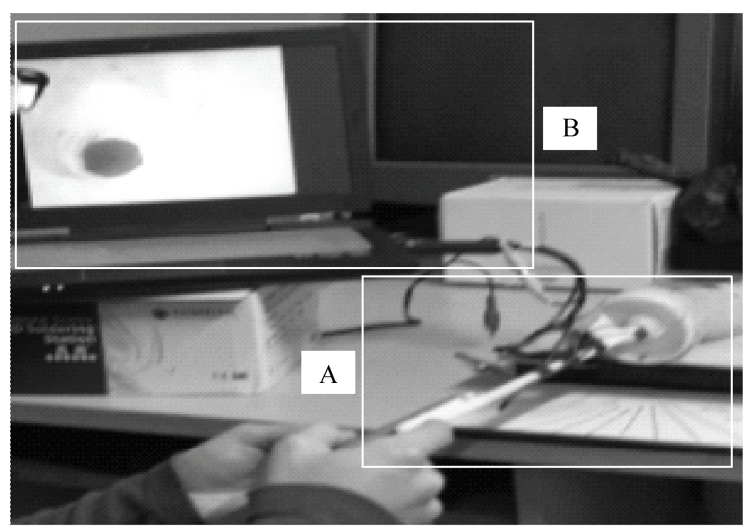

(a)

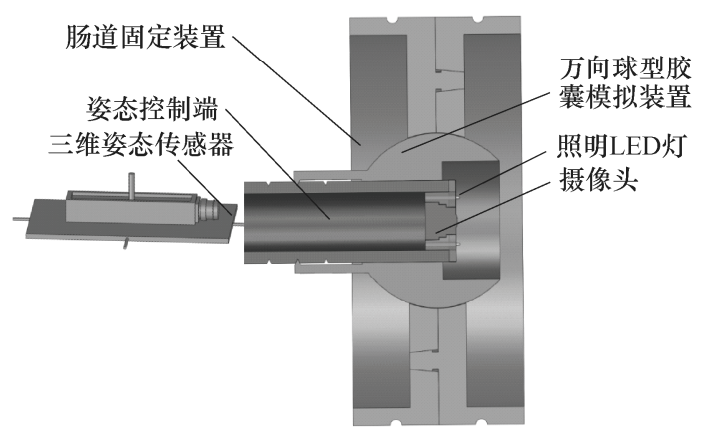

(b)

图 10 试验装置

试验时, 首先调整摄像机姿态, 记录下第一次 拍摄姿态信息, 提取此时图像暗区质心; 然后仅改 变侧摆角, 记录下第二次拍摄姿态信息, 提取此时 图像暗区质心，通过两幅图像计算导航方向；最后 保持自转角度固定, 调整侧摆角和俯仰角使暗区处
于图像正中心, 记录此时姿态信息, 并与之前计算 的导航方向对比。以上步骤为一组试验数据, 如 图 11a 所示。在试验过程中分别测试了肠道内壁较 多褶皱和较少褶皱两种情况，以试验此方法对肠壁 褶皱的抗干扰性。提取试验所拍摄图像的暗区质心， 部分试验图像如图 11b 所示, 图 11 中黑色暗区中间 像素点即为计算所得图像暗区质心。

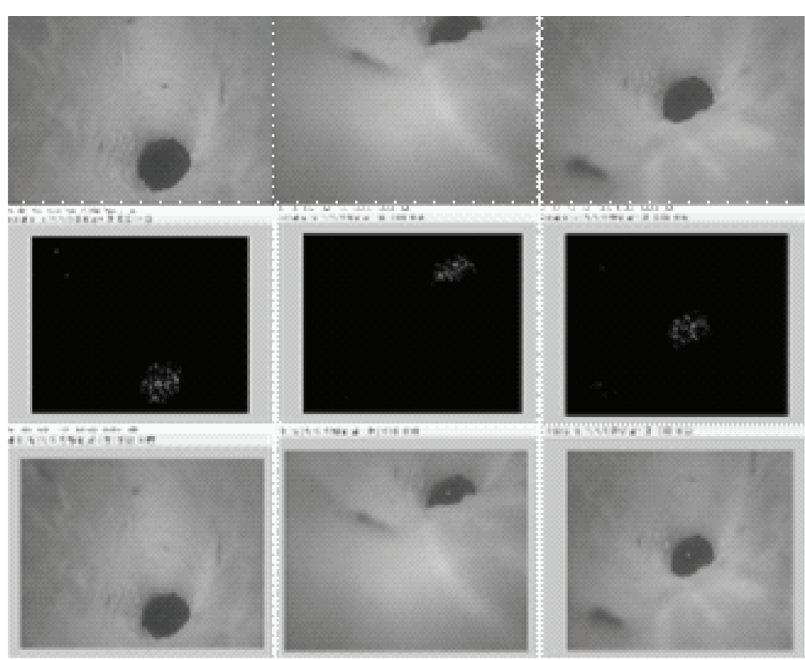

(a)
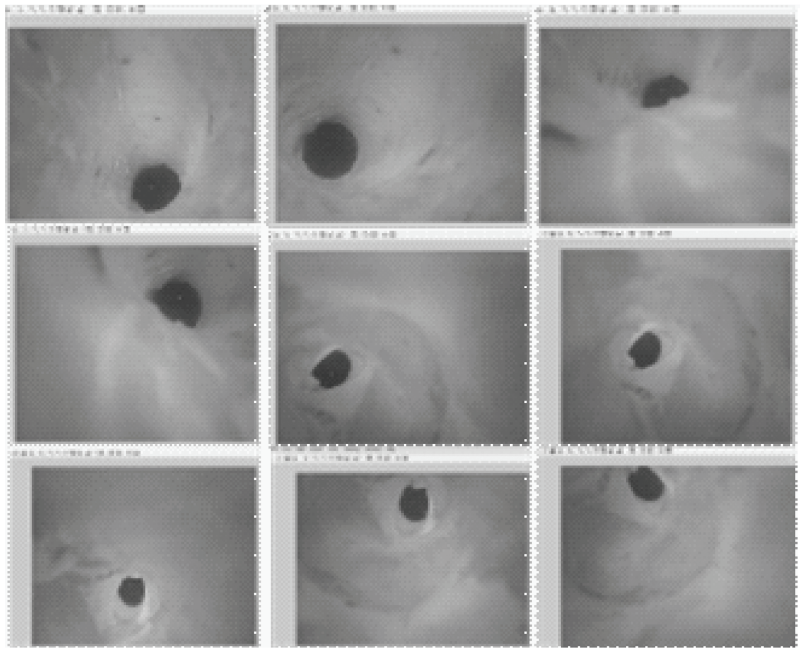

(b)

图 11 部分试验图像

具体试验数据如表 2 所示。通过试验发现计 算结果与真实值有一定误差, 其中试验误差数据 如表 3 所示。自转角的测量误差值在 $5^{\circ}$ 以内, 确定 导航方向的俯仰角和侧摆角的误差值在 $1^{\circ} \sim 2^{\circ}$, 由 于双半球型胶囊滚动具有容错性, 这个误差范围可以 实现弯曲肠道中对双半球型胶囊机器人进行导航。

其中影响导航中方向角的误差来源主要包括磁 矢量方位误差、机器人与胃肠内壁摩擦带来的误差、 暗区质心计算误差和图像识别误差等。通过该试验 证明了本文所提出导航方法的有效性, 为双半球型 胶囊机器人的导航研究奠定了基础。 


\section{表 2 试验数据表}

\begin{tabular}{ccccc}
\hline 组数 & $\begin{array}{c}\text { 第一次拍摄 } \\
\theta, \delta, \alpha \text { 值 } /\left(^{\circ}\right)\end{array}$ & $\begin{array}{c}\text { 第二次拍摄 } \\
\theta, \delta, \alpha \text { 值 } /\left(^{\circ}\right)\end{array}$ & $\begin{array}{c}\alpha^{*} \text { 计算值 } \\
/\left(^{\circ}\right)\end{array}$ & $\begin{array}{c}\text { 导航方向 } \\
\text { 计算值 } /\left(^{\circ}\right)\end{array}$ \\
\hline 1 & $10,0,-95$ & $30,0,-95$ & -98.27 & $(26.74,0.82)$ \\
2 & $0,0,-65$ & $30,0,-65$ & -70.85 & $(22.74,4.69)$ \\
3 & $20,6,45$ & $-3,6,45$ & 47.40 & $(7.24,-4.16)$ \\
4 & $0,-10,-45$ & $-9,-10,-45$ & -42.74 & $(-9.31,-6.13)$ \\
5 & $6,-10,-95$ & $-20,-10,-95$ & -100.20 & $(-9.23,-4.09)$ \\
6 & $-20,-10,-135$ & $10,-10,-135$ & -139.93 & $(-9.56,-6.22)$ \\
\hline
\end{tabular}

注: $1 \sim 3$ 组为肠壁褶皱较多环境下数据, $4 \sim 6$ 组为肠壁褶皱较少环境 下数据。

表 3 试验误差数据表

\begin{tabular}{cccc}
\hline 组数 & $\begin{array}{c}\alpha^{*} \text { 计算误差值 } \\
/\left(^{\circ}\right)\end{array}$ & $\begin{array}{c}\text { 导航方向真 } \\
\text { 实值 } /\left(^{\circ}\right)\end{array}$ & $\begin{array}{c}\text { 导航方向误差值 } \\
/\left(^{\circ}\right)\end{array}$ \\
\hline 1 & 3.27 & $(25,0)$ & $(1.74,0.82)$ \\
2 & 5.85 & $(21,3)$ & $(1.74,1.69)$ \\
3 & 2.40 & $(8,-5)$ & $(0.76,0.84)$ \\
4 & 2.26 & $(-8,-5)$ & $(1.31,1.13)$ \\
5 & 5.20 & $(-8,-5)$ & $(1.23,0.91)$ \\
6 & 4.93 & $(-8,-5)$ & $(1.56,1.22)$ \\
误差平均值 & 3.96 & - & $(1.39,1.10)$ \\
\hline
\end{tabular}

\section{6 结论}

为了实现双半球型胶囊在弯曲肠道内转弯滚 动, 根据双半球型胶囊轴线与空间万向旋转磁场轴 线的同轴随动特性, 本文提出一种单目视觉辅助导 航方法, 该方法是以同一俯仰角和两个不同侧摆角 下单目摄像头所拍摄的弯曲肠道内部两幅无线传输 图像为基础, 通过计算弯曲肠道图像暗区质心的方 位来确定胶囊的视觉导航方位。

单目视觉辅助导航方法不需安装任何传感器、 对硬件系统依赖度低、安装精度要求低、抗电磁干 扰能力强、操纵便捷可靠, 通过离体猪大肠试验证 明了该方法的可行性。该导航方法为实现胶囊在肠 道复杂弯曲环境内的诊疗与定点靶向治疗等临床应 用奠定了基础。今后, 应在万向旋转磁场控制精度 和胶囊机器人姿态最优控制方法方面加强研究, 降 低胶囊稳定性对导航精度的影响, 此外, 还要加强 导航算法研究, 减小计算误差, 为实用化铺平道路。

\section{参 考 文 献}

[1] CHAN J, CHAO A, CHEUNG V, et al. Gastrointestinal disease burden and mortality: A public hospital-based study from 2005 to 2014[J]. Journal of Gastroenterology and Hepatology，2018，34(1): 124-131.

[2] CARAVACA A M, PIESTUN R. Single multimode fiber
endoscope[J]. Optics Express, 2017, 25(3): 1656-1665.

[3] PARK S, PARK J, PARK H, et al. Multi-functional capsule rndoscope for gastro-intestinal tract[C]// International Joint International on Sice-icase. IEEE, 2006: 2090-2093.

[4] KIM S H, ISHIYAMA K. Magnetic robot and manipulation for active-locomotion with targeted drug release[J]. Mechatronics IEEE/ASME Transactions on, 2014, 19(5): 1651-1659.

[5] METTLER L, CLEVIN L, TERNAMiAN A, et al. The past, present and future of minimally invasive endoscopy in gynecology: A review and speculative outlook $[\mathrm{J}]$. Minimally Invasive Therapy \& Allied Technologies, 2013, 22(4): 210-226.

[6] LIEN G S, WU M S, CHEN C N, et al. Feasibility and safety of a novel magnetic-assisted capsule endoscope system in a preliminary examination for upper gastrointestinal tract[J]. Surgical Endoscopy, 2017, 32(4): 1937-1944.

[7] ZHANG Y S, ZICHUN Y U, YANG H Y, et al. Orthogonal transformation operation theorem of a spatial universal uniform rotating magnetic field and its application in capsule endoscopy[J]. Science China, 2017, 60(6): 854-864.

[8] 张永顺, 周华涛, 张林霞, 等. 一种新型双半球形胶囊 机器人[J]. 机械工程学报, 2017, 53(15): 110-118.

ZHANG Yongshun, ZHOU Huatao, ZHANG Linxia, et al. A new kind of dual hemisphere capsule robot[J]. Journal of Mechanical Engineering, 2017, 53(15): 110-118.

[9] 张永顺, 苏忠㑆, 杨振强, 等. 一种主被动双半球形 胶囊机器人及其姿态调整与转弯驱动控制方法: 中国, CN104983385A[P]. 2015-10-21.

ZHANG Yongshun, SU Zhongkan, YANG Zhenqiang, et al. An active/passive dual hemisphere capsule robot and its control strategy for posture adjustment and steering driving: China, CN104983385A[P]. 2015-10-21.

[10] MATEEN H, BASAR R, AHMED A U, et al. Localization of wireless capsule endoscope: A systematic review[J]. IEEE Sensors Journal, 2017， 17(5): 1197-1206.

[11] CHENG C, CHAI W, ROTH H. A single frame depth visual gyroscope and its integration for robot navigation and mapping in structured indoor environments[J]. Journal of Intelligent \& Robotic Systems, 2015, 80(3-4): 365-374.

[12] MING X, KONG D, YE L, et al. A new localization 
system for tracking capsule endoscope robot based on digital 3-Axis magnetic sensors array[C]// Proceedings of 2017 Chinese Intelligent Systems Conference. 2017: 487-494.

[13] WEISS R, ITZKE A, REITENSPIESS J, et al. A novel closed loop current sensor based on a circular array of magnetic field sensors[J]. IEEE Sensors Journal, 2018, 99: 1.

[14] 赵昕扬. 用于手术导航的高精度三目视觉定位方法研 究[D]. 南京: 南京理工大学, 2017.

ZHAO Xinyang. Research on high precision trinocular vision positioning method for surgical navigation[D]. Nanjing: Nanjing University of Technology, 2017.

[15] LUO X, JAYARATHNE U L, PAUTLER S E, et al. Binocular endoscopic 3-D scene reconstruction using color and gradient-boosted aggregation dtereo matching for robotic surgery[C]// International Conference on Image and Graphics. Springer, Cham, 2015: 664-676.

[16] WANG D, LIU H, CHENG X. A miniature binocular endoscope with local feature matching and dtereo matching for $3 \mathrm{D}$ measurement and $3 \mathrm{D}$ reconstruction[J]. Sensors, 2018, 18(7): 2243-2263.

[17] TURAN M, PILAVCI Y Y, GANIYUSUFOGLU I, et al. Sparse-then-dense alignment-based 3D map recons- truction method for endoscopic capsule robots[J]. Machine Vision \& Applications, 2018, 29(2): 345-359.

[18］赵荧. 基于视觉的胶囊内窥镜导航研究[D]. 哈尔滨: 哈 尔滨工业大学, 2013.

ZHAO Ying. Vision-based capsule endoscope navigation research[D]. Harbin: Harbin Institute of Technology, 2013.

[19] RAMAN B, KUMAR S, ROY P P, et al. Proceedings of international conference on computer vision and image processing[M]. Singapore: Springer, 2017.

[20] SAGNIKA S, BILGAIYAN S, MISHRA B S P. Workflow scheduling in cloud computing environment using bat algorithm[J]. International Journal of Computer Applications, 2018, 89(2): 11-18.

[21] 于子淳. 基于独立变量的空间万向旋转磁场正交变换 操作原理[D]. 大连: 大连理工大学, 2017.

YU Zichun. Principle of orthogonal transformation of spatial universal rotating magnetic field based on independent variables[D]. Dalian: Dalian University of Technology, 2017.

作者简介: 张永顺(通信作者), 男, 1965 年出生, 博士, 教授, 博士研 究生导师。主要研究方向为机器人机电一体化技术等。

E-mail: zyshun@dlut.edu.cn

田丰, 男, 1993 年出生, 硕士研究生。主要研究方向为双半球型胶囊机 器人导航。

E-mail: 416804156@qq.com 